\title{
FLUID VELOCITY FIELDS DERIVED FROM VORTICITY SINGULARITIES
}

\author{
BY \\ K. B. RANGER \\ Department of Mathematics, University of Toronto, Toronto, Ontario, Canada M5S $3 G 3$
}

\begin{abstract}
The present article addresses the existence by construction of random velocity fields produced by prescribed flow singularities which are consistent with the equations governing the motion of a two-dimensional viscous incompressible liquid. By showing first that the vorticity can be arbitrary and consistent with the flow equations it is then possible to determine the velocity field explicitly in terms of the vorticity and its derivatives.

The methods described here are also applicable to axisymmetric motion and also fully three-dimensional flow.
\end{abstract}

Introduction. In their relatively early review of the fluid dynamic literature, Dryden, Murnaghan, and Bateman [1] describe in one section the manner in which a twodimensional viscous fluid behaves when the vorticity distribution is prescribed as a function of the independent variables. The analysis is not pursued to the point where the velocity field components are explicitly derived in terms of the vorticity distribution, and it is not shown the extent to which the vorticity can be prescribed. In order to circumvent this problem it is required that a certain determinant should vanish, and from this analysis it is possible to predict various classes of Navier-Stokes solutions which are viscosity dependent. Some of these solutions for the stream function $\psi$ were previously derived by Jeffrey [2] in a different way. In general, these solutions relate to the situation in which lines of constant vorticity coincide with one family of coordinate curves. For example, in polar coordinates $(r, \theta)$, there is a solution for the stream function $\psi$ where the lines of constant vorticity coincide with the family of circles $r=$ positive constant, and is expressed by

$$
\psi=-b \nu \theta+A_{0} r^{b+2}+B_{0} r^{2}+C_{0} \ln r, b \neq-2
$$

with $A_{0}, B_{0}, C_{0}, b$ arbitrary constants. There is also a solution for the special case $b \neq-2$. The flow represents a viscosity dependent solution of the Navier-Stokes equations where the lines of constant vorticity coincide with the family of concentric circles $r=$ constant.

Received June, 2003.

2000 Mathematics Subject Classification. Primary 76Dxx, 76D17. 
The stream function is of some interest since the arbitrary constants can be chosen in order to describe the steady motion of a circular cylinder rotating with constant angular velocity $w_{0}$, in an infinite fluid at rest at infinity. In addition there is uniform suction at the boundary with constant normal velocity. If the suction velocity is $-Q \hat{r}$, where $q_{r}=-\frac{1}{r} \psi_{\theta}=\frac{b \nu}{a}=-Q$, at the boundary $r=a$, then the appropriate stream function satisfying $q_{\theta}=\psi_{r}=w_{0} a$ at $r=a$, and $|\underline{q}| \rightarrow 0, r \rightarrow \infty$ is expressed by

$$
\psi=Q_{a} \theta+A_{0} r^{2-\frac{a Q}{\nu}}+\left[w a^{2}-A_{0}\left(2-\frac{a Q}{\nu}\right) a^{2-\frac{a Q}{\nu}}\right] \ln r
$$

where $A_{0}$ is an arbitrary constant. The constant $B_{0}$ vanishes in order to maintain a single valued pressure field. The fluid velocity vanishes at large distances provided that $\frac{a Q}{\nu}>2$, and the stream function $\psi$ represents one of the simpler straightforward examples of non-uniqueness for two-dimensional viscous flow when $\nu<\frac{a Q}{2}$, since the constant $A_{0}$ is arbitrary. The condition $2 \nu<a Q$ is consistent with the general results concerning uniqueness of viscous flow described by Leray [3] and Serrin [4]. It is also noted that the example cited is sufficiently elementary to be included as an exercise in the book by Acheson [5].

It is observed that the boundary conditions

$$
\begin{aligned}
\int_{0}^{2 \pi} q_{r} r d \theta & =-2 \nu \pi, \\
\int_{0}^{2 \pi} q_{\theta} r d \theta & =I \text { at } r=a, \\
\frac{\partial q_{r}}{\partial \theta} & =0, \text { as } r \rightarrow 0, \\
\left(q_{r} \cdot q_{\theta}\right) & \rightarrow 0, \quad r \rightarrow \infty
\end{aligned}
$$

where

$$
q_{r}=-\frac{1}{r} \frac{\partial \psi}{\partial \theta}, \quad q_{\theta}=\frac{\partial \psi}{\partial r}
$$

are sufficient to determine a unique solution of the Stokes flow equation

$$
\nabla^{4} \psi=0, \quad \nabla^{2}=\frac{\partial^{2}}{\partial r^{2}}+\frac{1}{r} \frac{\partial}{\partial r}+\frac{1}{r^{2}} \frac{\partial^{2}}{\partial \theta^{2}},
$$

and the solution is given by

$$
\psi=2 \nu \theta+\frac{I}{2 \pi} \ln r,
$$

which incidentally also satisfies $\nabla^{2} \psi=0$. In the case of the Navier-Stokes equations, there is a solution satisfying the same boundary conditions and is given by

$$
\psi=2 \nu \theta+A_{0}(\ln r)^{2}+\ln r\left[\frac{I}{2 \pi}-2 A_{0} \ln a\right] .
$$

and the constant $A_{0}$ is arbitrary, indicating a lack of uniqueness which is not viscosity dependent. In the case of singularities as forcing flow it follows that the non-uniqueness does not depend necessarily on an inequality involving the kinematic viscosity. The resulting motion is not random as the normal component of velocity is prescribed. 
Before stating the objective in the present article it is of interest to discuss a methodological analogue in the theory of elementary differential equations. Consider the extended Clairaut equation defined by

$$
y=p x+f(p), \quad p=\frac{d y}{d x}
$$

where $f(p)$ is an arbitrary differentiable function. By differentiation it follows that

$$
p=p+\left[x+f^{\prime}(p)\right] \frac{d p}{d x},
$$

in which case

$$
x=-f^{\prime}(p) \text { or } p=c, \text { a constant. }
$$

The singular solution is described in parametric form by

$$
x=-f^{\prime}(p) . \quad y=f(p)-p f^{\prime}(p),
$$

and the sccond solution is represented by

$$
y=c x+f(c) .
$$

It is known from the literature that the solutions for arbitrary $c$ constitute the family of tangent lines to the envelope curve defined by the singular solution. However, in the present context there is a point of interest which is obvious but not directly pointed out, and this is that the singular solution, albeit in parametric form, is determined entirely by differentiation of the basic non-linear equation and requires no integration procedure. In any event for a given $f(p)$ the solution can be graphed in the $(x, y)$ plane.

The fact that solutions can be constructed entircly by differentiation from certain differential equations forms the basis of generalization to partial differential equations, and in particular the Navier-Stokes equations for the motion of a viscous incompressible liquid. In the ensuing analysis, which is straightforward, it is shown that by iterated differentiation of the momentum equation maintaining the vorticity as an arbitrary function then by applications of consistency conditions leads to the conclusion that the vorticity is undetermined and arbitrary. This in turn implies that the fluid velocity can be constructed in terms of an arbitrary vorticity distribution subject to certain provisions. The analysis involved in this procedure is unavoidably cumbersome but the final results for the velocity field in terms of the arbitrary vorticity distribution are reasonably compact especially in the case of inviscid flow $(\nu \rightarrow 0)$. The analysis is simplified considerably by the use of complex variable notation for two-dimensional flow. These results are applicable to the motion produced by forced singularities in the vorticity distribution which represents a substantial generalization of the results depicted by the Jeffrey solution. The application of the results to boundary value and initial value problems is out of reach because there is no guarantee that the velocity field is sufficiently general to satisfy initial or boundary conditions.

These results can be generalized to the situation of axial symmetry, and can be extended further to fully three-dimensional motion. In general for this situation the vorticity possesses two arbitrary components and flow produced by singularities is of a more general nature. 
1. The equations of motion. The descriptive equations for the motion of a viscous incompressible liquid can be written as

$$
\begin{aligned}
& \underline{q}_{t}+[\operatorname{curl} \underline{q} \times \underline{q}]=-\underline{\nabla} H+\nu \nabla^{2} \underline{q} \\
& H=p / \rho_{0}+\frac{1}{2}|\underline{q}|^{2}, \quad \operatorname{div} \underline{q}=0
\end{aligned}
$$

where $q$ is the fluid velocity, $H$ the Bernoulli function, $p$ the pressure, $\rho_{0}$ the constant density, and $\nu$ the kinematic viscosity.

For two-dimensional motion the velocity field can be described in terms of a stream function $\psi=\psi(x, y, t)$ and

$$
\underline{q}=\operatorname{curl}(-\psi \hat{k})=-\psi_{y} \hat{i}+\psi_{x} \hat{j} .
$$

In this case the component equations of (1) are expressed by

$$
\begin{aligned}
-\psi_{y t}-\psi_{x} \nabla^{2} \psi & =-H_{x}-\nu \frac{\partial}{\partial y} \nabla^{2} \psi \\
\psi_{x t}-\psi_{y} \nabla^{2} \psi & =-H_{y}+\nu \frac{\partial}{\partial x} \nabla^{2} \psi .
\end{aligned}
$$

In complex variable notation $z=x+i y, \bar{z}=x-i y$, it follows that

$$
2 \frac{\partial}{\partial z}=\frac{\partial}{\partial x}-i \frac{\partial}{\partial y}, \quad 2 \frac{\partial}{\partial \bar{z}}=\frac{\partial}{\partial x}+i \frac{\partial}{\partial y} .
$$

and the complex flow equation is expressed by $(4)+i(5)$, which simplifies to

$$
i \psi_{\bar{z} t}-4 \psi_{\bar{z}} \psi_{z \bar{z}}=-H_{\bar{z}}+4 \nu i \psi_{z \overline{z z}} .
$$

If $w=4 \psi_{z \bar{z}}$, then by elimination of $H$ it is found that

$$
\psi_{z} w_{\bar{z}}-\psi_{\bar{z}} w_{z}=2 i \nu w_{z \bar{z}}-\frac{i}{2} w_{t} .
$$

Also by differentiation of (7) with respect to $z$ and taking the real part it is found that

$$
2 H_{z \bar{z}}=4 \frac{\partial}{\partial z}\left(\psi_{\bar{z}} \psi_{z \bar{z}}\right)+4 \frac{\partial}{\partial \bar{z}}\left(\psi_{z} \psi_{z \bar{z}}\right)
$$

and by formal integration $H$ is expressed by

$$
H=2 \iint\left[\frac{\partial}{\partial z}\left(\psi_{\bar{z}} \psi_{z \bar{z}}\right)+\frac{\partial}{\partial \bar{z}}\left(\psi_{z} \psi_{z \bar{z}}\right)\right] d z d \bar{z} .
$$

In the next section an application of the vorticity transport equation will be discussed in connection with the random motion of a viscous fluid.

2. Construction of the fluid velocity field. To construct the fluid velocity field it is initially appropriate to consider the system of steady state equations

$$
\begin{aligned}
\psi_{z} w_{\bar{z}}-\psi_{\bar{z}} w_{z} & =2 i \nu w_{z \bar{z}} \\
\Omega & =4 \psi_{z \bar{z}}^{\prime}
\end{aligned}
$$

where $w, \Omega$ are for the present independent arbitrary real functions of $(x, y)$. The results of this section are also applicable to time dependent motion where $w_{z \bar{z}}$ is replaced by $w_{z \bar{z}}-\frac{1}{4} w_{t}$. 
The objective in this analysis is to show by repeated application of consistency conditions to the basic flow equations (11), (12) that the vorticity $\Omega$ is essentially undetermined. As a consequence of this result it is possible to prescribe the fluid velocity in a general manner in terms of the vorticity and its derivatives. In turn the vorticity is essentially arbitrary. This result arises from the fact that the consistency conditions lead to four identities in the derivatives of $w$. These identities are a feature of the structure of the basic vorticity equation and the definition of the vorticity distribution. The analysis predicting this conclusion is unavoidably cumbersome, but the results for the velocity distribution are reasonably compact in terms of complex variable notation.

In the first instance it is necessary to demonstrate existence of solutions for $\psi$ by formal construction from the real variable form of equation (11) provided by

$$
\psi_{x} w_{y}-\psi_{y} w_{x}=\nu \nabla^{2} w
$$

If $\chi$ is a real function of $(x, y)$ such that $(w, \chi)$ is a one-one mapping into $(x, y)$ then

$$
w_{x}=\frac{y \chi}{x_{w} y_{\chi}-x_{\chi} y_{w}}, \quad w_{y}=\frac{-x \chi}{x_{w} y_{\chi}-x_{\chi} y_{w}}
$$

and

$$
x_{w} y_{\chi}-x_{\chi} y_{w}=\frac{1}{w_{x} \chi_{y}-w_{y} \chi_{x}} \neq 0 .
$$

In this case equation (15) can be written as

$$
-\psi_{\chi}=\frac{\nu \nabla^{2} w}{w_{x} \chi_{y}-w_{y} \chi_{x}}
$$

and integration shows that

$$
\psi=\int \frac{\nu \nabla^{2} w d \chi}{\left(w_{y} \chi_{x}-w_{x} \chi_{y}\right)}+g(\psi)
$$

and

$$
\Omega=4 \frac{\partial^{2}}{\partial z \partial \bar{z}}\left\{\int \frac{\nu \nabla^{2} w d \chi}{\left(w_{y} \chi_{x}-w_{x} \chi_{y}\right)}+g(\psi)\right\} .
$$

It follows by this formal integration that solutions for the velocity $q=2 i \psi_{\bar{z}}$ can be constructed in which $w$ is arbitrary.

Returning to the complex variable form given by (11) it is found that $\psi_{\bar{z}}$ is expressed by

$$
\psi_{\bar{z}}=\psi_{z} \frac{w_{\bar{z}}}{w_{z}}-\frac{2 i \nu w_{z \bar{z}}}{w_{z}}
$$

and by differentiation with respect to $\bar{z}$ it is found that

$$
\psi_{\overline{z z}}=\frac{1}{4} \Omega \frac{w_{\bar{z}}}{w_{z}}+\psi_{z} \frac{\partial}{\partial \bar{z}}\left(\frac{w_{\bar{z}}}{w_{z}}\right)-2 i \nu \frac{\partial}{\partial \bar{z}}\left(\frac{w_{z \bar{z}}}{w_{z}}\right),
$$

for which the complex conjugate equation is

$$
\psi_{z z}=\frac{1}{4} \Omega \frac{w_{z}}{w_{\bar{z}}}+\psi_{\bar{z}} \frac{\partial}{\partial z}\left(\frac{w_{z}}{w_{\bar{z}}}\right)+2 i \nu \frac{\partial}{\partial z}\left(\frac{w_{z \bar{z}}}{w_{\bar{z}}}\right) .
$$


A further differentiation of $(20)$ with respect to $\bar{z}$ produces the equation

$$
\begin{aligned}
\frac{1}{4} \Omega_{\bar{z}}= & \frac{1}{4} \frac{\partial}{\partial z}\left(\Omega \frac{w_{\bar{z}}}{w_{z}}\right)-2 i \nu \frac{\partial^{2}}{\partial z \partial \bar{z}}\left(\frac{w_{z \bar{z}}}{w_{z}}\right)+\psi_{z} \frac{\partial^{2}}{\partial z \partial \bar{z}}\left(\frac{w_{\bar{z}}}{w_{z}}\right) \\
& +\frac{\partial}{\partial \bar{z}}\left(\frac{w_{\bar{z}}}{w_{z}}\right)\left[\frac{1}{4} \Omega \frac{w_{z}}{w_{\bar{z}}}+\psi^{\prime} \bar{z} \frac{\partial}{\partial z}\left(\frac{w_{z}^{\prime}}{w_{\bar{z}}^{\prime}}\right)+2 i \nu \frac{\partial}{\partial z}\left(\frac{w_{z \bar{z}}}{w \bar{z}}\right)\right],
\end{aligned}
$$

with the aid of (21). Equation (22) can be expressed in the form

$$
\frac{1}{4} w_{z} \Omega_{\bar{z}}-\frac{1}{4} w_{\bar{z}} \Omega_{z}-a_{0} \Omega=b_{0} \psi_{z}+c_{0} \psi_{\bar{z}}+d_{0} .
$$

where the coefficient functions $a_{0}, b_{0}, c_{0}, d_{0}$ are defined by

$$
\begin{aligned}
& a_{0}=\frac{1}{4}\left[w_{z} \frac{\partial}{\partial z}\left(\frac{w_{\bar{z}}}{w_{z}}\right)+\frac{w_{z}^{2}}{w_{\bar{z}}} \frac{\partial}{\partial \bar{z}}\left(\frac{w_{\bar{z}}}{w_{z}}\right)\right]=\frac{w_{z}^{2} w_{\bar{z} z}-w_{\bar{z}}^{2} w_{z z}}{4 w_{z} w_{\bar{z}}}, \\
& b_{0}=w_{z} \frac{\partial^{2}}{\partial z \partial \bar{z}}\left(\frac{w_{\bar{z}}}{w_{z}}\right), \quad c_{0}=w_{z} \frac{\partial}{\partial \bar{z}}\left(\frac{w_{\bar{z}}}{w_{z}}\right) \frac{\partial}{\partial z}\left(\frac{w_{z}}{w_{\bar{z}}}\right), \\
& d_{0}=2 i \nu w_{z} \frac{\partial}{\partial \bar{z}}\left(\frac{w_{\bar{z}}}{w_{z}}\right) \frac{\partial}{\partial z}\left(\frac{w_{z z}}{w_{\bar{z}}}\right)-2 i \nu w_{z} \frac{\partial^{2}}{\partial z \partial \bar{z}}\left(\frac{w_{z \bar{z}}}{w_{z}}\right) .
\end{aligned}
$$

The real and imaginary parts of equation (22) are expressed by

$$
\left(b_{0}+\bar{c}_{0}\right) \psi_{z}+\left(\bar{b}_{0}+c_{0}\right) \psi_{\bar{z}}+d_{0}+\bar{d}_{0}=0,
$$

and

$$
\frac{1}{2}\left(w_{z} \Omega_{\bar{z}}-w_{\bar{z}} \Omega_{z}\right)-\left(a_{0}-\bar{a}_{0}\right) \Omega=\left(b_{0}-\bar{c}_{0}\right) \psi_{z}-\left(\bar{b}_{0}-c_{0}\right) \psi_{\bar{z}}+d_{0}-\bar{d}_{0} .
$$

With the use of the equation

$$
\psi_{z} w_{\bar{z}}-\psi_{\bar{z}} w_{z}=2 i \nu w_{z} \bar{z}
$$

equation (27) can be written as

$$
\left[\left(b_{0}+\bar{c}_{0}\right) w_{z}+\left(\bar{b}_{0}+c_{0}\right) w_{\bar{z}}\right] \psi_{\bar{z}}+2 i \nu\left(b_{0}+\bar{c}_{0}\right) w_{z \bar{z}}+\left(d_{0}+\bar{d}_{0}\right) w_{\bar{z}}=0 .
$$

It is readily verified without providing the straightforward details that

$$
\begin{aligned}
w_{z}\left(b_{0}+\bar{c}_{0}\right)+w_{\bar{z}}\left(\bar{b}_{0}+c_{0}\right)= & w_{z}\left[w_{z} \frac{\partial^{2}}{\partial z \partial \bar{z}}\left(\frac{w_{\bar{z}}}{w_{z}}\right)+w_{z} \frac{\partial}{\partial \bar{z}}\left(\frac{w_{\bar{z}}}{w_{z}}\right) \frac{\partial}{\partial z}\left(\frac{w_{z}}{w_{\bar{z}}}\right)\right] \\
& +w_{\bar{z}}\left[w_{\bar{z}} \partial^{2} z \partial \bar{z}\left(\frac{w_{z}}{w_{\bar{z}}}\right)+w_{z} \frac{\partial}{\partial \bar{z}}\left(\frac{w_{\bar{z}}}{w_{z}}\right) \frac{\partial}{\partial z}\left(\frac{w_{z}}{u_{\bar{z}}^{\prime}}\right)\right]=0
\end{aligned}
$$


and

$$
\begin{aligned}
2 i \nu( & b_{0} \\
+ & \left.\bar{c}_{0}-\bar{b}_{0}-c_{0}\right) w_{z \bar{z}} \\
& +\left(d_{0}+\bar{d}_{0}\right)\left(w_{z}+w_{\bar{z}}\right) \\
= & 2 i \nu\left[w_{z} \frac{\partial^{2}}{\partial z \partial \bar{z}}\left(\frac{w_{\bar{z}}}{w_{z}}\right)-w_{\bar{z}} \frac{\partial^{2}}{\partial z \partial \bar{z}}\left(\frac{w_{z}}{w_{\bar{z}}}\right)+\left(w_{\bar{z}}-w_{z}\right) \frac{\partial}{\partial z}\left(\frac{w_{z}}{w_{\bar{z}}}\right) \frac{\partial}{\partial \bar{z}}\left(\frac{w_{\bar{z}}}{w_{z}}\right)\right] \\
& +\left(w_{z}+w_{\bar{z}}\right)\left[2 i \nu w_{z} \frac{\partial}{\partial \bar{z}}\left(\frac{w_{\bar{z}}}{w_{z}}\right) \frac{\partial}{\partial z}\left(\frac{w_{z \bar{z}}}{w_{\bar{z}}}\right)-2 i \nu w_{z} \frac{\partial^{2}}{\partial z \partial \bar{z}}\left(\frac{w_{z \bar{z}}}{w_{z}}\right)\right. \\
& \left.-2 i \nu w_{\bar{z}} \frac{\partial}{\partial z}\left(\frac{w_{z}}{w_{\bar{z}}}\right) \frac{\partial}{\partial \bar{z}}\left(\frac{w_{z \bar{z}}}{w_{z}}\right)+2 i \nu w_{\bar{z}} \frac{\partial^{2}}{\partial z \partial \bar{z}}\left(\frac{w_{z \bar{z}}}{w_{\bar{z}}}\right)\right] \\
= & 0
\end{aligned}
$$

are both identities in the derivatives of $w$, and therefore independent of $w$. These identities result from the basic structure of the basic equations (11), (12). It also follows that equation (23) can be written with contracted notation in the form

$$
A \psi_{z}-\bar{A} \psi_{\bar{z}}+B=0
$$

where

$$
\begin{gathered}
A=w_{z} \frac{\partial^{2}}{\partial z \partial \bar{z}}\left(\frac{w_{\bar{z}}}{w_{z}}\right)-w_{\bar{z}} \frac{\partial}{\partial z}\left(\frac{w_{z}}{w_{\bar{z}}}\right) \frac{\partial}{\partial \bar{z}}\left(\frac{w_{\bar{z}}}{w_{z}}\right) \\
B=\frac{1}{2} \Omega \frac{\left[w_{z}^{2} w_{\overline{z z}}-w_{\bar{z}}^{2} w_{z z}\right]}{w_{z} w_{\bar{z}}}-\frac{1}{2}\left(w_{z} \Omega_{\bar{z}}-w_{\bar{z}} \Omega_{z}\right) \\
2 i \nu\left\{w_{z} \frac{\partial}{\partial z}\left(\frac{w_{z \bar{z}}}{w_{\bar{z}}}\right) \frac{\partial}{\partial \bar{z}}\left(\frac{w_{\bar{z}}}{w_{z}}\right)+w_{\bar{z}} \frac{\partial}{\partial \bar{z}}\left(\frac{w_{z \bar{z}}}{w_{z}}\right) \frac{\partial}{\partial z}\left(\frac{w_{z}}{w_{\bar{z}}}\right)\right. \\
\left.\quad-w_{z} \frac{\partial^{2}}{\partial z \partial \bar{z}}\left(\frac{w_{z \bar{z}}}{w_{z}}\right)-w_{\bar{z}} \frac{\partial^{2}}{\partial z \partial \bar{z}}\left(\frac{w_{z \bar{z}}}{w_{z}}\right)\right\}
\end{gathered}
$$

so that $B+\bar{B}=0$. Again utilizing the equation

$$
\psi_{z} w_{\bar{z}}-\psi_{\bar{z}} w_{z}=2 i \nu w_{z \bar{z}}
$$

$\psi_{\bar{z}}$ can then be expressed as

$$
\psi_{\bar{z}}=\frac{\left[2 i \nu A w_{z \bar{z}}+B w_{\bar{z}}\right]}{\left[\bar{A} w_{\bar{z}}-A w_{z}\right]}
$$

and the complex fluid velocity $q=u+i v=2 i \psi_{\bar{z}}$, can be expressed in terms of a formula containing $\Omega$ and its derivatives, together with the derivatives of $w$. 
The explicit expression for $q$ is given below:

$$
\begin{aligned}
q= & \frac{i}{\left[w_{\bar{z}}^{2} \frac{\partial^{2}}{\partial z \partial \bar{z}}\left(\frac{w_{z}}{w_{\bar{z}}}\right)-w_{z}^{2} \frac{\partial^{2}}{\partial z \partial \bar{z}}\left(\frac{w_{\bar{z}}}{w_{z}}\right)\right]}\left\{2 i \nu\left[w_{z} \frac{\partial^{2}}{\partial z \partial \bar{z}}\left(\frac{w_{\bar{z}}}{w_{z}}\right)-w_{\bar{z}} \frac{\partial}{\partial z}\left(\frac{w_{z}}{w_{\bar{z}}}\right) \frac{\partial}{\partial \bar{z}}\left(\frac{w_{\bar{z}}}{w_{z}}\right)\right]\right. \\
& +\frac{1}{2 w_{z}} \Omega\left(w_{z}^{2} w_{\overline{z z}}-w_{\bar{z}}^{2} w_{z z}\right)-\frac{1}{2}\left(w_{z} \Omega_{\bar{z}}-w_{\bar{z}} \Omega_{z}\right) \\
& +2 i \nu\left[w_{z} \frac{\partial}{\partial z}\left(\frac{w_{z \bar{z}}}{w_{\bar{z}}}\right) \frac{\partial}{\partial \bar{z}}\left(\frac{w_{\bar{z}}}{w_{z}}\right)+w_{\bar{z}} \frac{\partial}{\partial \bar{z}}\left(\frac{w_{z \bar{z}}}{w_{z}}\right) \frac{\partial}{\partial z}\left(\frac{w_{z}}{w_{\bar{z}}}\right)\right. \\
& \left.\left.-w_{z} \frac{\partial^{2}}{\partial z \partial \bar{z}}\left(\frac{w_{z \bar{z}}}{w_{z}}\right)-w_{\bar{z}} \frac{\partial^{2}}{\partial z \partial \bar{z}}\left(\frac{w_{z \bar{z}}}{w_{\bar{z}}}\right)\right]\right\}
\end{aligned}
$$

This formula for $q$ represents an initial first step for the determination of the fluid velocity. To make further progress in this regard it is appropriate to write equation (23) in the form

$$
S+d \psi_{z}+\bar{d} \psi_{\bar{z}}=0
$$

where $S$ is real and

$$
S=a \Omega_{z}+\bar{a} \Omega_{\bar{z}}+b \Omega+c .
$$

The coefficients $a, b, c, d$ can be identified as

$$
\begin{aligned}
a= & \frac{1}{2} i w_{z}, \quad b= \\
c=i\left(\bar{d}_{0}-d_{0}\right)= & 2 \nu w_{\bar{z}} \frac{\partial}{\partial z}\left(\frac{w_{z}}{w_{\bar{z}}}\right) \frac{\partial}{\partial \bar{z}}\left(\frac{w_{z \bar{z}}}{w_{z}}\right)-2 \nu w_{\bar{z}} \frac{\partial^{2}}{\partial z \partial \bar{z}}\left(\frac{w_{z \bar{z}}}{w_{\bar{z}}}\right) \\
& +2 \nu w_{z} \frac{\partial}{\partial \bar{z}}\left(\frac{w_{\bar{z}}}{w_{z}}\right) \frac{\partial}{\partial z}\left(\frac{w_{z \bar{z}}}{w_{\bar{z}}}\right)-2 \nu w_{z} \frac{\partial^{2}}{\partial z \partial \bar{z}}\left(\frac{w_{z \bar{z}}}{w_{z}}\right) \\
d=i\left(\bar{c}_{0}-b_{0}\right)= & i\left[w_{\bar{z}} \frac{\partial}{\partial z}\left(\frac{w_{z}}{w_{\bar{z}}}\right) \frac{\partial}{\partial \bar{z}}\left(\frac{w_{\bar{z}}}{w_{z}}\right)-w_{z} \frac{\partial^{2}}{\partial z \partial \bar{z}}\left(\frac{w_{\bar{z}}}{w_{z}}\right)\right] .
\end{aligned}
$$

Once again with the use of the equation (11) and elimination of $\psi_{z}$ from (28) it is found that

$$
S a^{\prime}+b^{\prime}+\psi_{\bar{z}}=0
$$

where

$$
\begin{aligned}
a^{\prime} & =\frac{w_{\bar{z}}}{d w_{z}+\bar{d} w_{\bar{z}}}=\frac{i w_{\bar{z}}}{\left[w_{\bar{z}}^{2} \frac{\partial^{2}}{\partial z \partial \bar{z}}\left(\frac{w_{z}}{w_{\bar{z}}}\right)-w_{z}^{2} \frac{\partial^{2}}{\partial z \partial \bar{z}}\left(\frac{w_{\bar{z}}}{w_{z}}\right)\right]} \\
b^{\prime} & =\frac{2 \nu i w_{z \bar{z}}\left[w_{z} \frac{\partial^{2}}{\partial z \partial \bar{z}}\left(\frac{w_{\bar{z}}}{w_{z}}\right)-w_{\bar{z}} \frac{\partial}{\partial z}\left(\frac{w_{z}}{w_{\bar{z}}}\right) \frac{\partial}{\partial \bar{z}}\left(\frac{w_{\bar{z}}}{w_{z}}\right)\right]}{\left[w_{z}^{2} \frac{\partial^{2}}{\partial z \partial \bar{z}}\left(\frac{w_{\bar{z}}}{w_{z}}\right)-w_{\bar{z}}^{2} \frac{\partial^{2}}{\partial z \partial \bar{z}}\left(\frac{w_{z}}{w_{\bar{z}}}\right)\right]} .
\end{aligned}
$$

Differentiation of (44) with respect to $z$ provides the equation

$$
S_{z}+S \frac{a_{z}^{\prime}}{a^{\prime}}+\frac{b_{z}^{\prime}}{a^{\prime}}+\frac{1}{4 a^{\prime}} \Omega=0 .
$$

As a matter of interest equation (47) is of significance because $\Omega$ is real and satisfies a complex partial differential equation for which the real and imaginary parts are expressed 
by the equations

$$
\begin{aligned}
&\left(\frac{\partial}{\partial z}+\frac{\partial}{\partial \bar{z}}\right)\left\{a \Omega_{z}+\bar{a} \Omega_{\bar{z}}+b \Omega+c\right\}+\left(\frac{a_{z}^{\prime}}{a^{\prime}}+\frac{\bar{a}_{\bar{z}}^{\prime}}{\bar{a}^{\prime}}\right)\left\{a \Omega_{z}+\bar{a} \Omega_{\bar{z}}+b \Omega+c\right\} \\
&+\frac{b_{z}^{\prime}}{a^{\prime}}+\frac{\bar{b}_{\bar{z}}^{\prime}}{\bar{a}^{\prime}}+\frac{1}{4}\left(\frac{1}{a^{\prime}}-\frac{1}{\bar{a}^{\prime}}\right) \Omega=0
\end{aligned}
$$

and

$$
\begin{aligned}
\left(\frac{\partial}{\partial z}-\frac{\partial}{\partial \bar{z}}\right)\left\{a \Omega_{z}+\bar{a} \Omega_{\bar{z}}+b \Omega+c\right\}+\left(\frac{a_{z}^{\prime}}{a^{\prime}}-\frac{\bar{a}_{\bar{z}}^{\prime}}{\bar{a}^{\prime}}\right)\left\{a \Omega_{z}+\bar{a} \Omega_{\bar{z}}+b \Omega+c\right\} & \\
& +\left(\frac{b_{z}^{\prime}}{a^{\prime}}-\frac{\bar{b}_{\bar{z}}^{\prime}}{\bar{a}^{\prime}}\right)+\frac{1}{4}\left(\frac{1}{a^{\prime}}-\frac{1}{\bar{a}^{\prime}}\right) \Omega=0 .
\end{aligned}
$$

These equations are independent and basically result from the non-linear convection acceleration term in the equations of motion. At this point the procedure for generating consistency equations by partial differentiation can be continued to the point where the $\Omega$-derivatives can be eliminated from the system to determine a single real equation containing $\Omega$. A further differentiation of (47) with respect to $\bar{z}$ produces the equation

$$
S_{z \bar{z}}+S_{\bar{z}} \frac{a_{z}^{\prime}}{a^{\prime}}+S \frac{\partial}{\partial \bar{z}}\left(\frac{a_{z}^{\prime}}{a^{\prime}}\right)+\frac{\partial}{\partial \bar{z}}\left(\frac{b_{z}^{\prime}}{a^{\prime}}\right)+\frac{1}{4 a^{\prime}} \Omega_{\bar{z}}+\frac{\Omega}{4} \frac{\partial}{\partial \bar{z}}\left(\frac{1}{a^{\prime}}\right)=0
$$

Since $S_{z \bar{z}}$ is real then by forming the complex conjugate equation of (50) and elimination of $S_{z \bar{z}}$ it is found that

$$
\begin{aligned}
S_{\bar{z}} \frac{a_{z}^{\prime}}{a^{\prime}}-S_{z} \frac{\bar{a}_{\bar{z}}^{\prime}}{\bar{a}^{\prime}}+S\left[\frac{\partial}{\partial \bar{z}}\left(\frac{a_{z}^{\prime}}{a^{\prime}}\right)\right. & \left.-\frac{\partial}{\partial z}\left(\frac{\bar{a}_{\bar{z}}^{\prime}}{\bar{a}^{\prime}}\right)\right]+\frac{\partial}{\partial z}\left(\frac{b_{z}^{\prime}}{a^{\prime}}\right)-\frac{\partial}{\partial z}\left(\frac{\bar{b}_{\bar{z}}^{\prime}}{\bar{a}^{\prime}}\right) \\
& +\frac{1}{4 a^{\prime}} \Omega_{\bar{z}} \frac{1}{4 \bar{a}^{\prime}} \Omega_{z}+\frac{\Omega}{4}\left[\frac{\partial}{\partial \bar{z}}\left(\frac{1}{a^{\prime}}\right)-\frac{\partial}{\partial z}\left(\frac{1}{\bar{a}^{\prime}}\right)\right]=0 .
\end{aligned}
$$

$S_{z}, S_{\bar{z}}, S$ can be eliminated from (51) utilizing (47) (44) and this results in the single equation

$$
\begin{aligned}
\frac{\bar{a}_{\bar{z}}^{\prime}}{\bar{a}^{\prime}}\left(\frac{b_{z}^{\prime}}{a^{\prime}}+\frac{\Omega}{4 a^{\prime}}\right)- & \frac{a_{z}^{\prime}}{a^{\prime}}\left(\frac{\bar{b}_{\bar{z}}^{\prime}}{\bar{a}^{\prime}}+\frac{\Omega}{4 \bar{a}^{\prime}}\right)+\frac{\partial}{\partial \bar{z}}\left(\frac{b^{\prime} z}{z^{\prime}}\right)-\frac{\partial}{\partial z}\left(\frac{\bar{b}^{\prime}}{\bar{a}^{\prime}}\right) \\
+ & {\left[\frac{\partial}{\partial \bar{z}}\left(\frac{a_{z}^{\prime}}{a^{\prime}}\right)-\frac{\partial}{\partial z}\left(\frac{\bar{a}_{\bar{z}}^{\prime}}{\bar{a}^{\prime}}\right)\right]\left[a \Omega_{z}+\bar{a} \Omega_{\bar{z}}+b \Omega+c\right] } \\
& +\frac{1}{4 a^{\prime}} \Omega_{\bar{z}}-\frac{1}{4 \bar{a}^{\prime}} \Omega_{z}+\frac{\Omega}{4}\left[\frac{\partial}{\partial \bar{z}}\left(\frac{1}{a^{\prime}}\right)-\frac{\partial}{\partial z}\left(\frac{1}{\bar{a}^{\prime}}\right)\right]=0 .
\end{aligned}
$$

Again for the sake of symbolic brevity it is convenient to write this equation as

$$
A^{\prime} \Omega_{z}+\bar{A}^{\prime} \Omega_{\bar{z}}+B^{\prime} \Omega+C^{\prime}=0
$$


where $A^{\prime}$ is complex, and $B^{\prime}$. $C^{\prime}$ are real. These in turn are defined by

$$
\begin{aligned}
& i A^{\prime}=a \frac{\partial^{2}}{\partial z \partial \bar{z}}\left\{\ln \left(\frac{a^{\prime}}{\bar{a}^{\prime}}\right)\right\}-\frac{1}{4 \bar{a}^{\prime}} . \\
& i B^{\prime}=\frac{\bar{a}_{\bar{z}}^{\prime}-a_{z}^{\prime}}{a^{\prime} \bar{a}^{\prime}}+b \frac{\partial^{2}}{\partial z \partial \bar{z}}\left\{\ln \left(\frac{a^{\prime}}{\bar{a}^{\prime}}\right)\right\}+\frac{1}{4}\left(\frac{\bar{a}_{z}^{\prime}}{\bar{a}^{\prime 2}}-\frac{a_{\bar{z}}^{\prime}}{a^{\prime 2}}\right) \\
& i C^{\prime}=\frac{b_{z}^{\prime} \bar{a}_{\bar{z}}^{\prime}-\bar{b}_{\bar{z}}^{\prime} a_{z}^{\prime}}{a^{\prime} \bar{a}^{\prime}}+\frac{\partial}{\partial \bar{z}}\left(\frac{b_{z}^{\prime}}{a^{\prime}}\right)-\frac{\partial}{\partial z}\left(\frac{\bar{b}_{\bar{z}}^{\prime}}{\bar{a}^{\prime}}\right)+c \frac{\partial^{2}}{\partial z \partial \bar{z}}\left\{\ln \left(\frac{a^{\prime}}{\bar{a}^{\prime}}\right)\right\} .
\end{aligned}
$$

Differentiation of $(53)$ with respect to $z$ derives the equation

$$
A^{\prime} \Omega_{z z}+A_{z}^{\prime} \Omega_{z}+\bar{A}^{\prime} \Omega_{z \bar{z}}+\bar{A}_{z}^{\prime} \Omega_{\bar{z}}+B^{\prime} \Omega_{z}+B_{z}^{\prime} \Omega+C_{z}^{\prime}=0
$$

Now from equations (44) (47) it follows that by elimination of $S$ that

$$
\begin{aligned}
& a \Omega_{z z}+\Omega_{z}\left(a_{z}+b+\frac{a a_{z}^{\prime}}{a^{\prime}}\right)+\Omega_{z}\left(\bar{a}_{z}+\bar{a} \frac{a_{z}^{\prime}}{a^{\prime}}\right) \\
& +\bar{a} \Omega_{z z}+\Omega\left(\frac{b_{z}^{\prime}}{a^{\prime}}+\frac{b a_{z}^{\prime}}{a^{\prime}}+\frac{1}{4 a^{\prime}}\right)+c_{z}+\frac{c a_{z}^{\prime}}{a^{\prime}}+\frac{b_{z}^{\prime}}{a^{\prime}}=0 .
\end{aligned}
$$

Equations (57), (58) represent two independent complex differential equations for the derivatives of $\Omega$. By climination of $\Omega_{z z}$ it is found that

$$
\begin{aligned}
\Omega_{z \bar{z}}\left(\bar{a}-a \frac{\bar{A}^{\prime}}{A^{\prime}}\right)+ & \Omega_{z}\left[a_{z}+b+\frac{a a_{z}^{\prime}}{a^{\prime}}-a \frac{A_{z}^{\prime}}{A^{\prime}}-a \frac{B^{\prime}}{A^{\prime}}\right] \\
+ & \Omega_{z}\left[\bar{a}_{z}+\frac{a a_{z}^{\prime}}{z^{\prime}}-a \frac{\bar{A}_{z}^{\prime}}{A^{\prime}}\right]+\Omega\left[\frac{b_{z}^{\prime}}{a^{\prime}}+\frac{b a_{z}^{\prime}}{a^{\prime}}+\frac{1}{4 a^{\prime}}-\frac{a B_{z}^{\prime}}{A^{\prime}}\right] \\
& +c_{z}+\frac{c a_{z}^{\prime}}{a^{\prime}}+\frac{b_{z}^{\prime}}{a^{\prime}}-\frac{a c_{z}^{\prime}}{A^{\prime}}=0 .
\end{aligned}
$$

By eliminating $\Omega_{z \ddot{z}}$ which is real from this complex equation then the resulting equation in terms of contracted symbolic notation is expressed by

$$
A^{\prime \prime} \Omega_{z}+\bar{A}^{\prime \prime} \Omega_{\bar{z}}+B^{\prime \prime} \Omega+C^{\prime \prime}=0
$$

where $A^{\prime \prime}$ is complex and $B^{\prime \prime} . C^{\prime \prime}$ are both real. The coefficients $A^{\prime \prime}, B^{\prime \prime}, C^{\prime \prime}$ are expressed by

$$
\begin{aligned}
& i A^{\prime \prime}=A^{\prime}\left(a_{z}+b+\frac{a a_{z}^{\prime}}{a^{\prime}}\right)-a A_{z}^{\prime}-a B^{\prime}+\bar{A}^{\prime}\left(a_{\bar{z}}+\frac{a \bar{a}_{\bar{z}}^{\prime}}{\bar{a}^{\prime}}\right)-\bar{a} A_{\bar{z}}^{\prime} \\
& i B^{\prime \prime}=\frac{A^{\prime}}{a^{\prime}}\left(b_{z}^{\prime}+b a_{z}^{\prime}+\frac{1}{4}\right)-a B_{z}^{\prime}-\frac{\bar{A}^{\prime}}{\bar{a}^{\prime}}\left(\bar{b}_{\bar{z}}^{\prime}+b \bar{a}_{\bar{z}}^{\prime}+\frac{1}{4}\right)+\bar{a} \bar{B}_{\bar{z}}^{\prime} \\
& i C^{\prime \prime}=A^{\prime}\left[c_{z}+c \frac{a_{z}^{\prime}}{a^{\prime}}+\frac{b_{z}^{\prime}}{a^{\prime}}\right]-a c_{z}^{\prime}-\bar{A}\left[c_{\bar{z}}+\frac{c \bar{a}_{\bar{z}}^{\prime}}{\bar{a}^{\prime}}+\frac{\bar{b}_{\bar{z}}^{\prime}}{\bar{a}^{\prime}}\right]+\overline{a c_{\bar{z}}^{\prime}} .
\end{aligned}
$$

It is clear that the cocfficients $A^{\prime \prime}, B^{\prime \prime}, C^{\prime \prime}$ are different functions from $A^{\prime}, B^{\prime}, C^{\prime}$. because $A^{\prime \prime}$ ctc. contain derivatives of $a^{\prime}, b^{\prime}$ and $\frac{A^{\prime \prime}}{A^{\prime}} \neq \frac{B^{\prime \prime}}{B^{\prime}} \neq \frac{C^{\prime \prime}}{C^{\prime}}$. Also if this were not the case then (48) (49) would not be independent. In this case equations (53) (60) are independent 
even though they are basically derived from the same equation (11) with the aid of (12). By elimination of $\Omega_{\bar{z}}$ from (53) (60) it is found that

$$
\Omega_{z}=E \Omega+F
$$

where

$$
E=\frac{A^{\prime \prime} B^{\prime}-\bar{A}^{\prime} B^{\prime \prime}}{A^{\prime \prime} \bar{A}^{\prime}-\bar{A}^{\prime \prime} A^{\prime}} . \quad F=\frac{A^{\prime \prime} C^{\prime}-C^{\prime \prime} \bar{A}^{\prime}}{A^{\prime \prime} \bar{A}^{\prime}-\bar{A}^{\prime \prime} A^{\prime}} .
$$

By differentiation with respect to $\bar{z}$

$$
\begin{aligned}
\Omega_{z \bar{z}} & =E \Omega_{\bar{z}}+E_{\bar{z}} \Omega+F_{\bar{z}} \\
& =\bar{E} \Omega_{z}+\bar{E}_{z} \Omega+\bar{F}_{z}
\end{aligned}
$$

and eliminating $\Omega, \Omega_{\bar{z}}$ it is found that

$$
\begin{aligned}
\Omega_{z \bar{z}} & =E \bar{E} \Omega+E \bar{F}+E_{\bar{z}} \Omega+F_{\bar{z}} \\
& =E \bar{E} \Omega+\bar{E} F+\bar{E}_{z} \Omega+\bar{F}_{z} .
\end{aligned}
$$

The real and imaginary parts of this equation, noting that $\Omega_{z \bar{z}}$ is real, produce the real equations

$$
\Omega\left(\bar{E}_{z}-E_{\bar{z}}\right)=E \bar{F}-\bar{E} F+F_{\bar{z}}-\bar{F}_{z}
$$

and

Now if

$$
\Omega_{z \bar{z}}=E \bar{E} \Omega+\frac{1}{2}\left[E \bar{F}+F \bar{E}+F_{\bar{z}}+\bar{F}_{z}\right] .
$$

$$
\bar{E}_{z}-E_{\bar{z}} \neq 0, \quad E \bar{F}-F \bar{E}+F_{\bar{z}}-\bar{F}_{z} \neq 0
$$

then for consistency equation (64) can be written as

$$
\frac{\partial}{\partial z}\left\{\frac{E \bar{F}-F \bar{E}+F_{\bar{z}}-\bar{F}_{z}}{\bar{E}_{z}-E_{\bar{z}}}\right\}=E \frac{\left\{E \bar{F}-F \bar{E}+F_{\bar{z}}-\bar{F}_{z}\right\}}{\left(E_{\bar{z}}-E_{\bar{z}}\right)}+F .
$$

By formal integration of this complex equation it is found that

$$
E \bar{F}-F \bar{E}+F_{\bar{z}}-\bar{F}_{z}=\left(\bar{E}_{z}-E_{\bar{z}}\right)\left\{e^{\int E d z}\left[\int F e^{-\int E d z} d z+f(\bar{z})\right]\right\}
$$

also $E, F$ are defined in terms of $w$-derivatives and this complex equation in general places restrictions on $w$ which in turn is arbitrary. To avoid this contradiction the only consistent possibility is that equation (72) is an identity. Since the expression

$$
e^{\int E d z}\left[\int F e^{-\int E d z} d z+f(\bar{z})\right]
$$

is complex and does not vanish it follows that

$$
E \bar{F}-F \bar{E}+F_{\bar{z}}-\bar{F}_{z}=0, \quad \bar{E}_{z}-E_{\bar{z}}=0
$$

and for consistency are identities and not differential equations, without placing constraints on $w$. It follows that the equations represented by (74) are identities in the $w$-derivatives. This result can also be verified directly by working through the excessively cumbersome calculation. In this case $\Omega$ is undetermined by the analysis and is essentially arbitrary. It is noted that these results emanate from the basic equations (11) and (12). In order to be consistent with the Navier-Stokes equations it is permissible 
to choose $\Omega=w$ and $w$ is arbitrary. It then follows that there exist by construction a class of fluid velocity fields which are defined in terms of an essentially arbitrary vorticity distribution. This result appears to be new for a non-linear dynamical system such as the Navier-Stokes equations. The analysis described here concerns two-dimensional motion, but this can be extended to axisymmetric. fully three-dimensional flow and time dependent motion. Utilizing the result that

$$
w_{\bar{z}}^{2} \frac{\partial^{2}}{\partial z \partial \bar{z}}\left(\frac{w_{z}}{w_{\bar{z}}}\right)-w_{z}^{2} \frac{\partial^{2}}{\partial z \partial \bar{z}}\left(\frac{w_{\bar{z}}}{w_{z}}\right)=2 i w_{z} w_{\bar{z}} \frac{\partial^{2}}{\partial z \partial \bar{z}}\left\{\ln \frac{w_{z}^{\prime}}{u_{\bar{z}}}\right\}
$$

the formula $q$ for the complex fluid velocity is given by

$$
\begin{aligned}
q= & \frac{1}{2 w_{z} w_{\bar{z}} \frac{\partial^{2}}{\partial z \partial \bar{z}}\left\{\ln \left(\frac{w_{z}^{\prime}}{w_{\bar{z}}}\right)\right\}}\left\{2 i \nu\left[w_{z} \frac{\partial^{2}}{\partial z \partial \bar{z}}\left(\frac{w_{\bar{z}}}{w_{z}}\right)-w_{\bar{z}} \frac{\partial}{\partial \bar{z}}\left(\frac{w_{z}}{w_{\bar{z}}}\right) \frac{\partial}{\partial \bar{z}}\left(\frac{w_{\bar{z}}}{w_{z}}\right)\right]\right. \\
& +\frac{w}{2 w_{z}}\left(w_{z}^{2} w_{\overline{z z}}-w_{\bar{z}}^{2} w_{z z}\right)+2 i \nu\left[w_{z} \frac{\partial}{\partial z}\left(\frac{w_{z \bar{z}}}{w_{\bar{z}}}\right) \frac{\partial}{\partial \bar{z}}\left(\frac{w_{\bar{z}}}{w_{z}}\right)+w_{\bar{z}} \frac{\partial}{\partial \bar{z}}\left(\frac{w_{z \bar{z}}}{w_{z}}\right) .\right. \\
& \left.\left.\frac{\partial}{\partial z}\left(\frac{w_{z}}{w_{\bar{z}}}\right)-w_{z} \frac{\partial^{2}}{\partial z \partial \bar{z}}\left(\frac{w_{z \bar{z}}}{w_{z}}\right)-w_{\bar{z}} \frac{\partial^{2}}{\partial z \partial \bar{z}}\left(\frac{w_{z \bar{z}}}{w_{\bar{z}}}\right)\right]\right\}
\end{aligned}
$$

where $w$ is arbitrary to the extent that

$$
w_{z} \neq 0, \quad \frac{\partial^{2}}{\partial z \partial \bar{z}}\left\{\ln \left(\frac{w_{z}}{w_{\bar{z}}}\right)\right\} \neq 0 .
$$

In the case of inviscid flow where $\nu=0$, there is a simplification and

$$
q=\frac{w\left[w_{z}^{2} w_{\overline{z z}}-w_{\bar{z}}^{2} w_{z z}\right]}{4 w_{z}^{2} w_{\bar{z}} \frac{\partial^{2}}{\partial z \partial \bar{z}}\left\{\ln \left(\frac{w_{z}}{w_{z}}\right)\right\}} .
$$

The main application of the formula for the fluid velocity ficld in terms of the vorticity is to the motion produced by prescribed singularities. For example if there is a prescribed singularity for the vorticity at the origin then there is in general a singularity in the velocity field at the same point, and the only other requirements are that the motion should be finite and the fluid velocity decay to zero at infinity. Since the formula for the velocity field represents a class of solutions of the Navier-Stokes equations and not the totality of solutions there is no guarantee that the solution is sufficiently general to be adapted to either a boundary value or initial value problem.

Now if the vorticity is prescribed by

$$
w=\frac{F(\theta)}{r k}, \quad F(\theta)=\sum_{n=0}^{\infty} A_{n} \cos \left(n \theta+\alpha_{n}\right)
$$

where $n$ is an integer and $k>2$, then this distribution produces a singularity in the velocity field which is partially determined by the equation

$$
\psi_{r r}+\frac{1}{r} \psi_{r}+\frac{1}{r^{2}} \psi_{\theta \theta}=\sum_{n=0}^{\infty} \frac{A_{n} \cos \left(n \theta+\alpha_{n}\right)}{r^{k}}
$$

from which it is readily inferred that the singularity in the velocity field is $O\left(r^{1-k}\right)$ as $r \rightarrow 0$. The precise nature of this singularity is determined by the formula (76) and is 
subject to the constraints

$$
w_{z} \neq 0, \quad \frac{\partial^{2}}{\partial z \partial \bar{z}}\left\{\ln \left(\frac{w_{z}}{w_{\bar{z}}}\right)\right\} \neq 0 \text { for } r>0 .
$$

This result can be generalized by writing $w$ as

$$
w=\frac{F(\theta)}{r k}+w^{\prime}
$$

where $w^{\prime}$ is of $O(1)$ as $r \rightarrow 0, w^{\prime}=O\left(r^{-m}\right), m>k-1$ as $r \rightarrow \infty$ and $w^{\prime}$ is single valued in the region $0 \leq \theta \leq 2 \pi$. In addition it is supposed that the generalized form of $w$ is subject to the restrictions imposed by the constraints (81). It follows that these relatively mild restrictions result in degrees of latitude for the choice of $w^{\prime}$, and the motion produced by the prescribed singularity is basically arbitrary.

It also appears that there are global velocity fields which are finite everywhere but basically arbitrary and only subject to the requirements

$$
w_{z} \neq 0, \quad \frac{\partial^{2}}{\partial z \partial \bar{z}}\left\{\ln \left(\frac{w_{z}}{w_{\bar{z}}}\right)\right\} \neq 0
$$

everywhere. It is also assumed that $w$ decays at infinity sufficiently rapidly to ensure the velocity field is finite at large distances.

These results appear to be new for the two-dimensional Navier-Stokes equations.

3. Steady motion with axial symmetry. Let $(x, \rho, \theta)$ be cylindrical polar coordinates; then the fluid velocity $q$ for axisymmetric steady viscous incompressible flow can be prescribed in terms of a stream function $\psi=\psi(x, \rho)$ and

$$
\underline{q}=-\left[\underline{\nabla} \times \frac{\psi}{\rho} \hat{\theta}\right]=-\frac{1}{\rho} \psi_{\rho} \hat{k}+\frac{1}{\rho} \psi_{x} \hat{\rho} .
$$

With standard notation the vector momentum equation governing the flow is expressed by

$$
[\operatorname{curl} \underline{q} \times \underline{q}]=-\underline{\nabla} H+\nu \nabla^{2} \underline{q},
$$

where $H$ is the Bernoulli function or total head of pressure defined by

$$
H=\frac{p}{\rho_{0}}+\frac{1}{2}|q|^{2} .
$$

The component equations of (84) are given by

$$
\begin{aligned}
-\frac{1}{p^{2}} \psi_{z} L_{-1}(\psi) & =-H_{z}-\frac{\nu}{\rho} \frac{\partial}{\partial \rho} L_{-1}(\psi) \\
-\frac{1}{\rho^{2}} \psi_{\rho} L_{-1}(\psi) & =-H_{\rho}+\frac{\nu}{\rho} \frac{\partial}{\partial x} L_{-1}(\psi)
\end{aligned}
$$

where $L_{-1}$ is the Stokes operator defined by

$$
L_{-1}(\psi)=\psi_{x x}+\psi_{\rho \rho}-\frac{1}{\rho} \psi_{\rho}
$$


There is only one component of vorticity and this is orthogonal to the meridinal plane in which case $\underline{w}=\operatorname{curl} \underline{q}=w \hat{\theta}=\frac{L_{-1}(\psi) \hat{\theta}}{\rho}$. If $H$ is eliminated from (87) (88) then the vorticity transport equation is provided by

$$
\frac{1}{\rho} \frac{\partial(\psi \cdot W)}{\partial(x \cdot \rho)}=\nu L_{3}(W)=\nu\left(W_{x x}+W_{\rho \rho}+\frac{3}{\rho} W_{\rho}\right)
$$

and $W=\frac{L_{-1}(\psi)}{\rho^{2}}$. $W$ is sometimes referred to as the ring vorticity, or vorticity density from the axis.

If $z=x+i \rho, \bar{z}=x-i \rho$, then in complex variable notation

$$
\frac{\partial}{\partial x}=\frac{\partial}{\partial z}+\frac{\partial}{\partial \bar{z}} \cdot \quad \frac{\partial}{\partial \rho}=i \frac{\partial}{\partial z}-i \frac{\partial}{\partial \bar{z}}
$$

and

$$
x=\frac{1}{2}(z+\bar{z}), \quad \rho=\frac{i}{2}(\bar{z}-z)
$$

in which case $\psi, W$ satisfies the equations

$$
\begin{aligned}
\psi_{z} W_{\bar{z}}-\psi_{\bar{z}} W_{z} & =\nu(z-\bar{z}) W_{z \bar{z}}+\frac{3 \nu}{2}\left(W_{z}-W_{\bar{z}}\right) \\
\psi_{z \bar{z}}-\frac{1}{2(z-z)}\left(\psi_{z}-\psi_{\bar{z}}\right) & =\frac{-W^{\prime}(z-\bar{z})^{2}}{16}
\end{aligned}
$$

where $W, W^{\prime}$ are independent and arbitrary. By a process of differentiation with respect to $z, \bar{z}$, it is found that $W^{\prime}$ satisfies a complex differential equation. The analysis is similar to the procedure described in the previous section but with the added feature caused by the presence of the space variable $2 i \rho=z-\bar{z}$. The net result shows that $W^{\prime}$ is essentially undetermined and can be chosen freely such that $W^{\prime}=W$ with $W$ arbitrary. In this case the complex fluid velocity

$$
\underline{q}=u+i v=\frac{4}{\bar{z}-z} \psi \bar{z},
$$

can be constructed in terms of $W$ and its derivatives with $W$ arbitrary, and represents a class of velocity fields satisfying the Navier-Stokes equations. As in the two-dimensional case the velocity field is sufficiently general to predict the motion produced by forced singularities, but not sufficiently general to describe the flow arising from boundary value or initial value problems.

In respect of the choice of basic singularity to choose for the vorticity distribution there is a preferential solution of the Navier-Stokes equations provided by the round jet or point source of momentum [6]. In this solution the stream function $\psi$ is expressed by

$$
\begin{aligned}
\psi= & \frac{2 \nu\left(1-\beta^{2}\right)}{E+\beta} \\
& \frac{2 \nu \rho^{2}}{E\left(x^{2}+\rho^{2}\right)^{1 / 2}+x} \\
& \frac{-\nu(z-\bar{z})^{2}}{2 E \sqrt{z \bar{z}}+z+\bar{z}}
\end{aligned}
$$


where $E$ is a constant with $|E|>1$, and $(r, \theta), \beta=\cos \theta$, are spherical polar coordinates. The ring vorticity is given by

$$
W=\frac{4 \nu}{\left[E\left(x^{2}+\rho^{2}\right)^{1 / 2}+x\right]^{3}}=\frac{4 \nu}{\left[E \sqrt{z \bar{z}}+\frac{1}{2}(z+\bar{z})\right]^{3}} .
$$

It follows from the analysis that there is a more general fluid velocity field for which the ring vorticity is given by

$$
W=\frac{4 \nu}{\left[E \sqrt{z \bar{z}}+\frac{1}{2}(z+\bar{z})\right]^{3}}+W^{\prime \prime}
$$

where $W^{\prime \prime}$ is basically arbitrary to the extent that the motion is finite and $W^{\prime \prime}$ behaves in a suitable manner near the origin and decaying at large distances.

The explicit formula for the velocity field is not given here as it is excessively cumbersome with the complication of the independent variable. However it appears to be sufficient to infer its explicit existence and represents a generalization of two-dimensional motion.

3-dimensional motion. For three-dimensional motion the vorticity has three components $\underline{w}=\operatorname{curl} \underline{q}, \underline{q}=q_{j} \hat{x}_{j}$, but only two components are independent since $\operatorname{div} \underline{w}=0$. The analysis described in the previous sections is applicable but again excessively cumbersome. The final expressions for the fluid velocity ficld contain two arbitrary components of the vorticity distribution.

Conclusions. It has been shown in the previous sections that arbitrary velocity fields can be generated from the momentum equations for the motion of a viscous incompressible liquid. This is achieved by the somewhat unusual method of partial differentiation only and is applicable essentially to a nonlinear system and not to linear equations. The solution represents a class of solutions that are suitable for the description of flow produced by a prescribed singularity and are not sufficiently general to cope with a boundary value or initial value problem. These results appear to be new for a dynamical system such as the Navier-Stokes equations and provide some quantitative information concerning the random motion of a viscous incompressible liquid. The pressure field can be found once the velocity field is known. This article discusses a generalization of some previously known non-unique flows to random motion produced by singularities.

\section{REFERENCES}

[1] H. L. Dryden, F. P. Murnaghan and H. Bateman, Hydrodynamics, Dover Publications, Inc., 242- 244 (1956)

[2] G. B. Jeffrey, The two-dimensional stead motion of a viscous fluid, Phil. Mag. 29 (6), 445-455 (1915)

[3] J. Leray, Étude de diverses integrales non-lineaires et de quelques problems que pose l'Hydrodynamiques, Jour. de Math. Pures et Appl. 12, 1 (1933)

[4] J. Serrin, Mathematical principles of classical fluid mechanics, Handbuch der Physik VIII/I, Springer-Verlag, Berlin 125-263 (1959)

[5] D. J. Acheson, Elementary Fluid Dynamics, Oxford University Press, 53 (1959)

[6] G. Batchelor, An Introduction to Fluid Dynamics CUP, 205-208 (1967) 